\title{
Electric Stimulation Hyperthermia Relieves Inflammation via the Suppressor of Cytokine Signaling 3-Toll Like Receptor 4 Pathway in a Prostatitis Rat Model
}

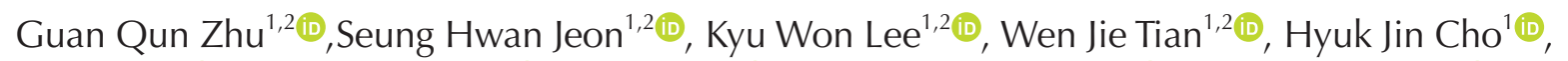

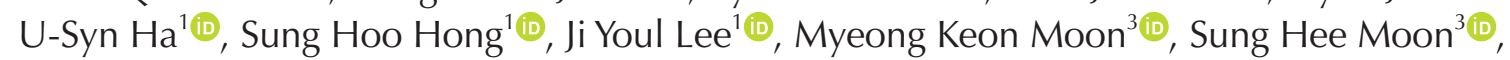
Sae Woong Kim ${ }^{1,2}$ (iD, Woong Jin Bae ${ }^{1,2(\mathbb{D})}$

${ }^{1}$ Department of Urology, College of Medicine, The Catholic University of Korea, ${ }^{2}$ Catholic Integrative Medicine Research Institute, The Catholic University of Korea, ${ }^{3}$ Buheung Medical, Seoul, Korea

Purpose: Chronic prostatitis (CP), including chronic pelvic pain syndrome (CPPS), is the most commonly encountered manifestation of prostatitis. The aim of this study was to evaluate the effect of electric stimulation hyperthermia treatment (ESHT) on CP/CPPS and to explore the underlying mechanism.

Materials and Methods: RWPE-2 cells with lipopolysaccharide-induced inflammation and a prostatitis rat model induced by $17 \beta$-estradiol and dihydrotestosterone underwent sham, electric stimulation, or ESHT treatment. Four weeks later, cells, supernatants, and rat prostates were collected for analysis using immunohistochemistry, Western blots, and enzyme-linked immunosorbent assays.

Results: We found that ESHT improved prostatitis in vivo and attenuated inflammation in vitro. ESHT significantly induced suppressor of cytokine signaling 3 (SOCS3) expression and subsequently promoted HSP70. It attenuated inflammation through decreased expression of toll-like receptor 4 (TLR4), nuclear factor kappa B, and subsequent inflammatory cytokines. ESHT also inhibited apoptosis and released growth factor in tissue affected by prostatitis.

Conclusions: ESHT improved CP/CPPS and reversed pathologic changes of prostatitis by inhibiting the SOCS3-TLR4 pathway.

Keywords: Electric stimulation hyperthermia treatment; Neuroinflammation; Prostatitis; Suppressor of cytokine signaling 3-toll like receptor 4 pathway

This is an Open Access article distributed under the terms of the Creative Commons Attribution Non-Commercial License (http://creativecommons.org/licenses/by-nc/4.0) which permits unrestricted non-commercial use, distribution, and reproduction in any medium, provided the original work is properly cited.

Received: May 16, 2019 Accepted: Jun 24, 2019 Published online Jul 23, 2019

Correspondence to: Woong Jin Bae iD https://orcid.org/0000-0001-7703-1161

Department of Urology, Seoul St. Mary's Hospital, College of Medicine, The Catholic University of Korea, 222 Banpo-daero, Seocho-gu, Seoul 06591, Korea.

Tel: +82-2-2258-6229, Fax: +82-2-2258-1536, E-mail: bwoong@catholic.ac.kr

Correspondence to: Sae Woong Kim (iD https://orcid.org/0000-0002-9127-9400

Department of Urology, Seoul St. Mary's Hospital, College of Medicine, The Catholic University of Korea, 222 Banpo-daero, Seocho-gu, Seoul 06591, Korea.

Tel: +82-2-2258-6226, Fax: +82-2-2258-1536, E-mail: ksw1227@catholic.ac.kr 


\section{INTRODUCTION}

Chronic prostatitis (CP), including chronic pelvic pain syndrome (CPPS), is the most commonly encountered manifestation of prostatitis, which is a highly prevalent syndrome with a significant negative impact on quality of life [1]. Both CP and CPPS are characterized by chronic pelvic pain. Little is known about the etiology of CP/CPPS, making it difficult to discover successful treatment therapies [2]. Wide-ranging research is being conducted to elucidate potential treatments, including single-agent therapies, multimodal therapies, botulinum toxin, immunomodulatory agents, and shock wave therapy [3]. However, there are insufficient data supporting the use of devices, and the exact mechanism of CP/CPPS has not been determined so far.

Wagenlehner et al [4] reported that patients with CP/CPPS suffered from perineal discomfort and pain, and that their pain developed through microglia-mediated neuroinflammation [5]. The stimulated microglia released various proinflammatory cytokines, including tumor necrosis factor-alpha (TNF- $\alpha$ ), interleukin-1 beta (IL-1B) and IL-6, resulting in a positive feedback loop that enhanced pain [6]. A recent study also showed that chronic pain was maintained partly by central sensitization controlled by neuroinflammation [7]. Similarly, neuroinflammation was found in a mouse model of prostatitis [8]. Therefore, we hypothesized that mitigating neuroinflammation would relieve pain in CP/CPPS.

Electric stimulation hyperthermia treatment (ESHT) has been used as a clinical intervention for many diseases, including tumors [9], pain [10], and diabetic angiopathy [11]. It is generally thought that ESHT influences cellular processes and functions [12], but there is still no consensus on the mechanism of ESHT.

In inflammation, toll-like receptor 4 (TLR4) is considered to be one of the most critical cytokines, and suppressor of cytokine signaling (SOCS) proteins function as inhibitors of TLR4 [13]. SOCS3, a member of the SOCS family, was observed to be an important negative regulator that suppresses IL- $1, \mathrm{TNF}-\alpha$, and IL-6 expression [14]. However, the role of SOCS3 in the TLR4 response remains controversial, as some studies have reported it to be a positive regulator [15], while others reported the opposite [16]. Therefore, in this study we explored the interaction between SOCS3 and TLR4 under ESHT stimulation and hypothesized that neuroinflammation would be relieved by inhibiting the
SOCS3-TLR4 pathway in prostatitis.

\section{MATERIALS AND METHODS}

\section{Ethics statement}

All animal experiments in this study were approved by the Institutional Animal Care and Use Committee of The Catholic University of Korea (CUMC-2016-021801). All surgery was performed under chloral hydrate anesthesia, and all efforts were made to minimize animals suffering.

\section{Cell preparation}

RWPE-2 cells (American Type Culture Collection [ATCC], Manassas, VA, USA) were cultured in low-glucose-containing Dulbecco's modified Eagle's medium (Gibco, Waltham, MA, USA) supplemented with $20 \%$ fetal bovine serum (Gibco) and $5 \mathrm{ng} / \mathrm{mL}$ basic fibroblast growth factor (Cell Signaling Technology, Danvers, MA, USA) at $37^{\circ} \mathrm{C}$ and $5 \% \mathrm{CO}_{2}$. Every 2 days, nonadherent cells were removed and fresh culture medium was added. Cells were passaged when they reached approximately $90 \%$ confluence.

\section{Electric stimulation hyperthermia treatment of cells in vitro}

The RWPE-2 cells were randomly divided into 4 groups: 1) a normal control group, 2) a lipopolysaccharide (LPS) group, 3) an electric stimulation (ES) group, and 4) an ESHT group. Cells in groups 2, 3, and 4 were treated with LPS $(10 \mu \mathrm{g} / \mathrm{mL})$. Cells in the ES group only received ES, while those in the ESHT group simultaneously received ES and hyperthermia. ESHT was performed using a medical ESHT device (Buheung Medical Co., Ltd., Seoul, Korea). The probe was kept in contact with the culture flask containing adherent cells covered with standard ultrasound gel. ES treatment was performed after cell attachment. Each generation of cell culture only received treatment once. Before ESHT and 12 hours after ESHT, cells and supernatants were collected and stored at $-80^{\circ} \mathrm{C}$ for analysis using enzyme-linked immunosorbent assays (ELISA) and Western blots.

\section{Experimental animal preparation and study design}

Eight-week-old male Sprague-Dawley rats (270-300

g) were purchased from a Korean company (Orient 
Bio Co., Seongnam, Korea). Rats ( $\mathrm{n}=40$ ) were randomly divided into 4 groups: 1) a sham group, 2) a prostatitis group, 3) an ES group, and 4) an ESHT group. Prostatitis was induced by $17 \beta$-estradiol and dihydrotestosterone for 4 weeks. After the prostatitis model was established, ES was administered to the rats in group 3, and ESHT to those in group 4.

\section{Electric stimulation hyperthermia treatment administration}

To administer ESHT, a medical device was used (Buheung Medical Co., Ltd.). This device had an electrofrequency stimulation in the range of 1 to $1,560 \mathrm{~Hz}$, a maximum hyperthermia treatment of $45^{\circ}$, and intensity-adjustable fine vibration; it consisted of a probe, a generator, and a component that generated direct heat when it was attached. Under anesthesia, the abdomen was shaved and exposed in a supine position. Ultrasound gel was applied to the abdomen, and then the ES applicator was placed on the abdomen. Rats in the ES group underwent ES and rats in the ESHT group underwent ES and hyperthermia. The treatment was administered every other day. After 4 weeks, the prostates were collected for analysis using immunohistochemistry, Western blots, and ELISA.

\section{Histology and immunohistochemistry}

The collected prostates were fixed in $4 \%$ paraformaldehyde for 24 hours at $4^{\circ} \mathrm{C}$ before being embedded in a paraffin block. The following primary antibodies were used: macrophage inflammatory protein $1 \alpha$ (MIP1 $\alpha$, diluted to 1:200; Abcam, Cambridge, UK), epidermal growth factor (EGF; diluted to 1:200; Santa Cruz Biotechnologies, Santa Cruz, CA, USA), caspase-3 (diluted to 1:200; Abcam), and 4,6-diamidino-2-phenylindole (DAPI; Vector Laboratories Inc., Burlingame, CA, USA). Digital images were obtained using a Zeiss LSM 510 Meta confocal microscope (Zeiss, Oberkochen, Germany), and the mean intensity was calculated using ZEN 2012 (Zeiss).

\section{Enzyme-linked immunosorbent assays and hematoxylin and eosin staining}

We quantified the expression of $\mathrm{LL}-1 \beta$, IL-6, and IL-8 by species-specific immunoassay ELISA kits (R\&D Systems Europe, Abingdon, UK) according to the manufacturer's instructions. Absorbance was read at a wavelength of $450 \mathrm{~nm}$ in a microplate reader (Synergy
H1 M; BioTek, Winooski, VT, USA). The prostates of each group were stained with hematoxylin and eosin (HE; Sigma, St. Louis, MO, USA) according to the manufacturer's instructions. After ESHT, prostates were collected and stored at $-80^{\circ} \mathrm{C}$ until use. Digital images were obtained using a Zeiss LSM 510 Meta confocal microscope (Zeiss).

\section{Apoptosis analysis in vitro and in vivo}

We assessed apoptosis via Western blotting for caspase-3 in vitro and by immunostaining for caspase-3 in vivo. After ESHT, cells and prostates from each group were collected and stored at $-80^{\circ} \mathrm{C}$ until use for apoptosis analysis.

\section{Western blotting}

The collected cells and tissues were homogenized using ice-cold radioimmunoprecipitation assay buffer (Cell Signaling Technology) containing an ethylenediaminetetraacetic acid-free protease inhibitor cocktail and phosphatase inhibitor cocktail (Roche Diagnostics $\mathrm{GmbH}$, Basel, Switzerland) and particulate mass was removed by centrifugation $(15,000 \times g)$ for 15 minutes at $4^{\circ} \mathrm{C}$. The supernatants were analyzed by sodium dodecyl sulfate-polyacrylamide gel electrophoresis. The primary antibodies used included HSP70 (diluted to 1:500; Abcam), SOCS3 (diluted to 1:200; Abcam), TLR4 (diluted to 1:200; Abcam), nuclear factor kappa B (NF$\kappa \mathrm{B})$ (diluted to 1:500; Abcam), $\beta$-actin (diluted to 1:1000; Abcam), caspase-3 (diluted to 1:200; Abcam), and inducible nitric oxide synthase (iNOS, diluted to 1:200; Santa Cruz Biotechnologies). The resulting images were analyzed using ImageJ (National Institutes of Health, Bethesda, MD, USA) to determine the integrated density of each protein band.

\section{Statistical analysis}

All data are presented as mean \pm standard error and were analyzed using IBM SPSS Statistics ver. 22.0 (IBM Corp., Armonk, NY, USA). The Student t-test, one-way analysis of variance (ANOVA), and $2 \times 2$ factorial ANOVA were used as appropriate to evaluate whether differences among groups were significant. p-values $<0.05$ were considered to indicate statistical significance. 


\section{RESULTS}

\section{Electric stimulation hyperthermia treatment improved prostatitis in vivo and attenuated inflammation in vitro}

To investigate the effect of ESHT on prostatitis, $\mathrm{HE}$ staining was performed. As shown in Fig. 1A, inflammation in the ESHT group was clearly improved compared to other groups. Compare with the prostatitis group, after ESHT, the epithelial layer was thinner and fewer papillary fronds protruded into the glandular cavities. We detected inflammatory factors, such as IL-1 $\beta$ in vivo and IL-6 and IL-8 in vitro using ELISA. As shown in Fig. 1B, after ESHT or ES, IL-1 $\beta$ expression decreased compared with the prostatitis group $(p<0.01)$, and in the ESHT group $\mathbb{L}-1 \beta$ decreased more than in the ES group (p<0.01). Fig. 1C and 1D show $\mathbb{L}-6$ and IL-8 expression before and after treatment in each group. Cells in the ESHT group expressed less IL-6 and IL-8 after treatment $(\mathrm{p}<0.01)$. Similarly, expression of IL-6 and IL-8 after ESHT was higher than in the LPS and ES groups $(p<0.01)$. Additionally, we found an interesting difference between the in vivo and in vitro analysis. The ES approach seemed to malfunction in vitro, whereas in vivo ES treatments still reduced inflammatory factors. A possible explanation for this observation is that the flask may have prevented the electric current from reaching the cells.

\section{A}

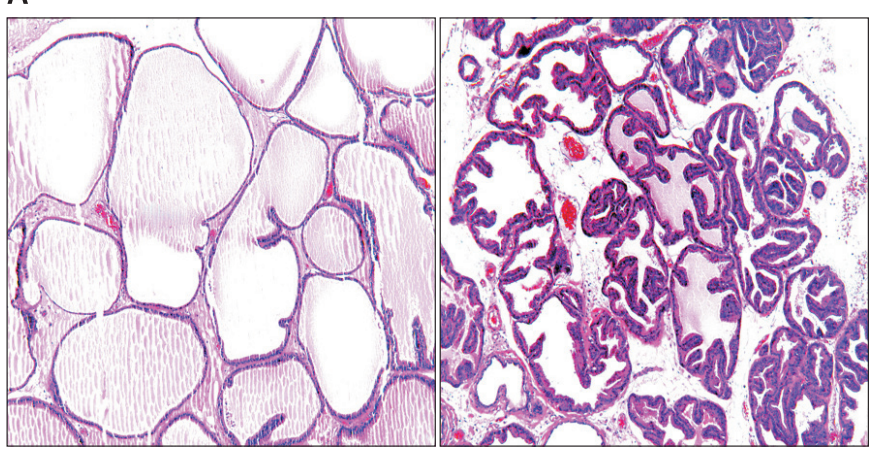

Sham

Prostatitis

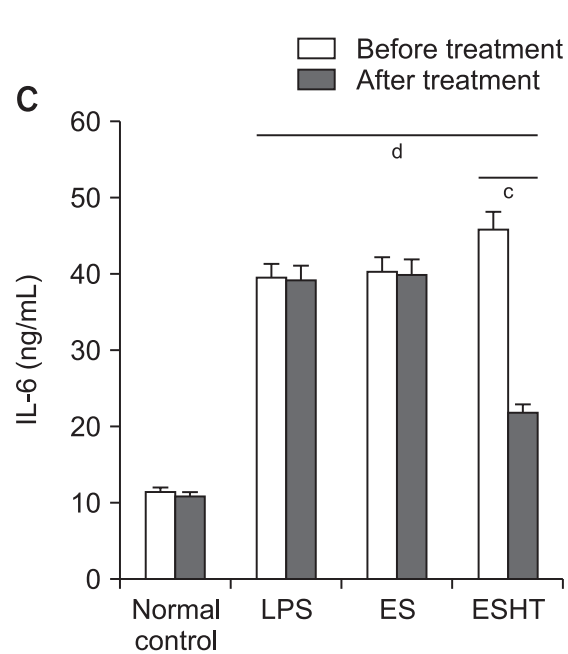

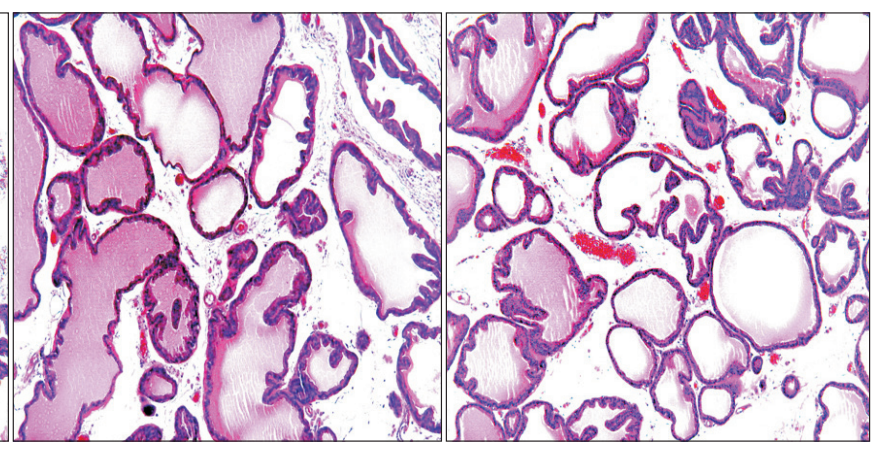

ES

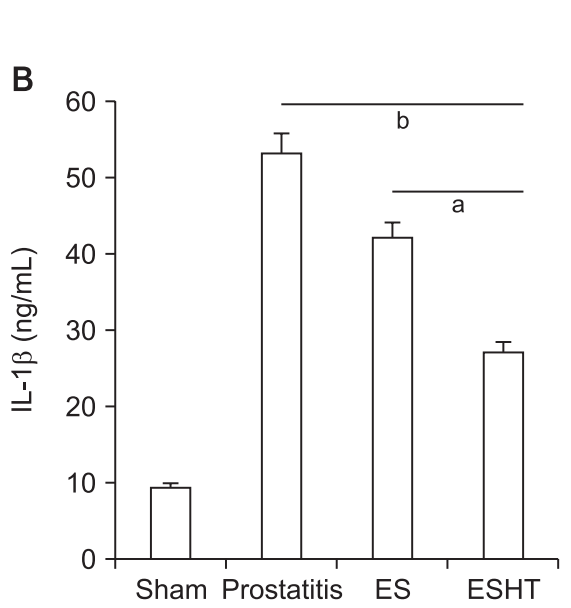
control

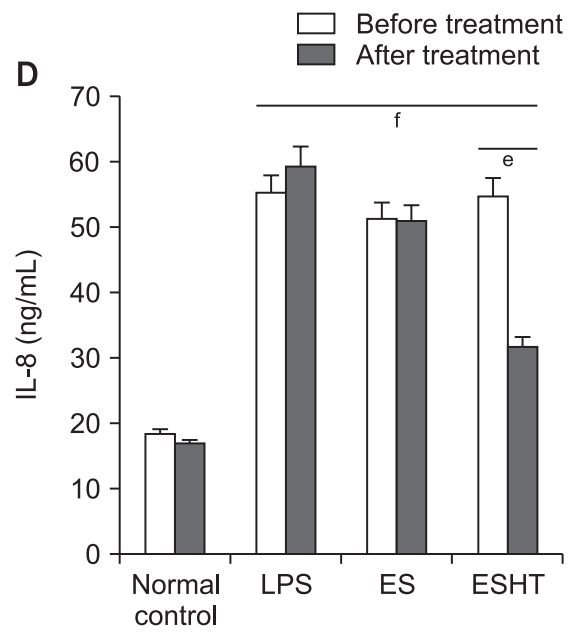

Fig. 1. Electric stimulation hyperthermia treatment (ESHT) improved prostatitis in vivo and attenuated inflammation in vitro. (A) Representative images of hematoxylin and eosin staining in the prostate for each group. Compared to the sham group, in the prostatitis group, the epithelial layer thickened and papillary fronds protruded into the glandular cavities simultaneously. After ESHT, prostatitis had clearly improved ( $\times 200)$. (B) Interleukin (IL)-1 $\beta$ concentrations in the prostate for each group after treatment, tested by enzyme-linked immunosorbent assay (ELISA). ${ }^{\mathrm{a}} \mathrm{p}<0.01$ compared to the electric stimulation (ES) group, ${ }^{\mathrm{b}} \mathrm{p}<0.01$ compared to the prostatitis group. (C) IL- 6 and (D) IL- 8 concentrations in vitro before and

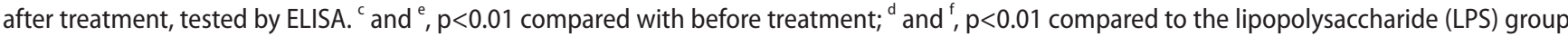
and ES group. 
A

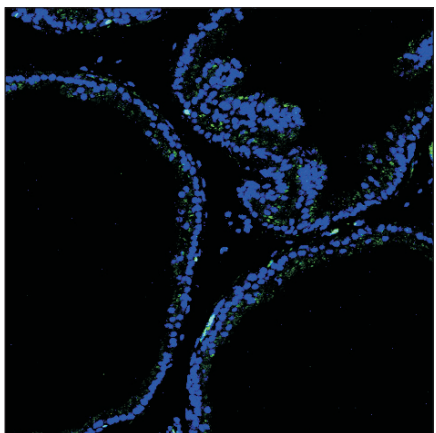

Sham

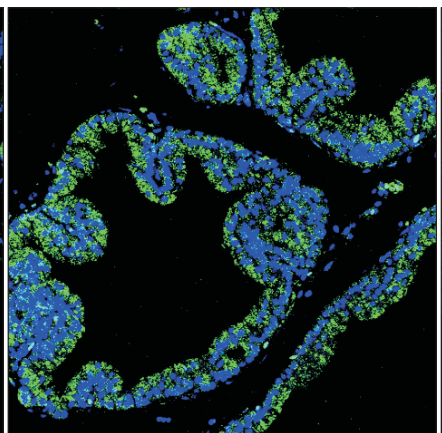

Prostatitis

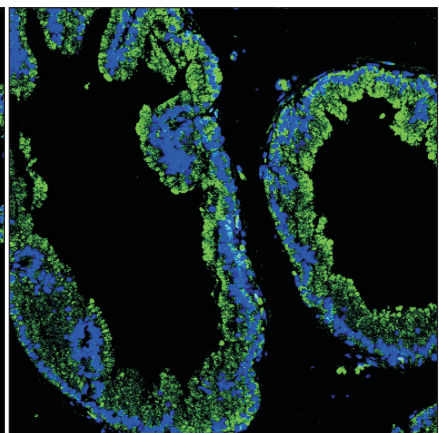

ES

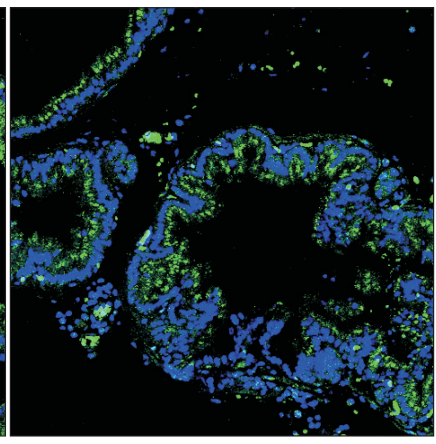

ESHT

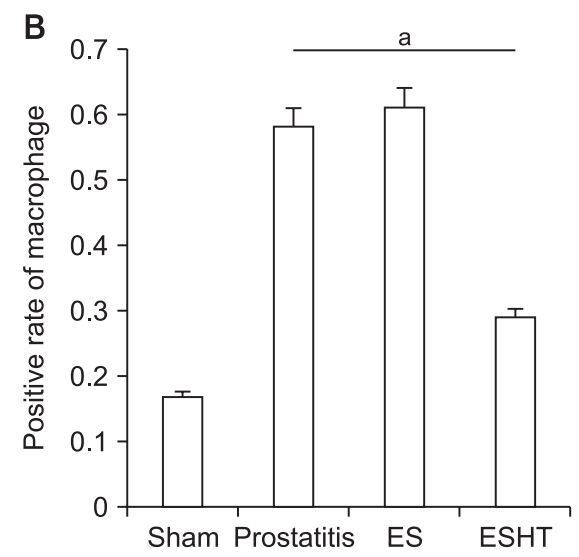

\section{Electric stimulation hyperthermia treatment decreased the number of macrophages in prostatitis}

Next, we investigated the amount of macrophages present in the prostatitis model by immunofluorescence of macrophages marked by MIP1 $\alpha$. These inflammatory cells were detected in the prostate in each group. Fig. 2A shows qualitatively that fewer macrophages were present in the prostate in the ESHT group. Fig. $2 \mathrm{~B}$ shows the quantitative results of macrophages in each group, demonstrating that there were fewer macrophages in the ESHT group than in the prostatitis and ES groups $(p<0.01)$. The reduction of macrophages by ESHT indicates that inflammation in prostate was alleviated after ESHT.

\section{Electric stimulation hyperthermia} treatment inhibited the toll-like receptor 4/nuclear factor kappa B pathway by stimulating suppressor or cytokine signaling 3 and increased HSP70 expression After we found that ESHT could improve prostatitis
Fig. 2. Electric stimulation hyperthermia treatment (ESHT) decreased the number of macrophages in the prostatitis model. (A) Representative images of macrophage staining in the prostate for each group. Green is MIP1 $\alpha$, blue is DAPI. (B) Quantitative positive rate of macrophages in vivo. MIP1 $\alpha$ : macrophage inflammatory protein $1 \alpha, \mathrm{DAPI}$ : 4,6-diamidino-2-phenylindole, ES: electric stimulation. ${ }^{\mathrm{a}} \mathrm{p}<0.01 \mathrm{com}-$ pared to the prostatitis group and ES group.

and relieve inflammation, we sought to characterize the underlying mechanism. To do so, protein expression was analyzed using Western blots, as shown in Fig. 3A and 3C. We found that after ESHT, inflammatory inhibitors including HSP70 and SOCS3 were upregulated, and inflammation-associated proteins including TLR4 and NF- $\mathrm{kB}$ were downregulated. Fig. 3B and $3 \mathrm{D}$ show the integrated density for each protein band, demonstrating that HSP70 and SOCS3 expression in the ESHT group was higher than in other groups $(p<0.01)$ and that TLR4 and NF- $\mathrm{BB}$ expression in the ESHT group was lower than in the other groups $(\mathrm{p}<0.01)$.

\section{Apoptosis was inhibited by electric stimulation hyperthermia treatment}

Caspase-3 expression was analyzed to detect apoptosis. As shown in Fig. 4A, after ESHT, caspase-3 expression in the prostate decreased. Fig. $4 \mathrm{~B}$ presents quantitative results for caspase-3 in vivo, showing lower levels of expression in the ESHT group than in other groups $(p<0.01)$. In vitro, caspase-3 expression showed similar results, as presented in Fig. $4 \mathrm{C}$ and $4 \mathrm{D}(\mathrm{p}<0.05)$. 
A

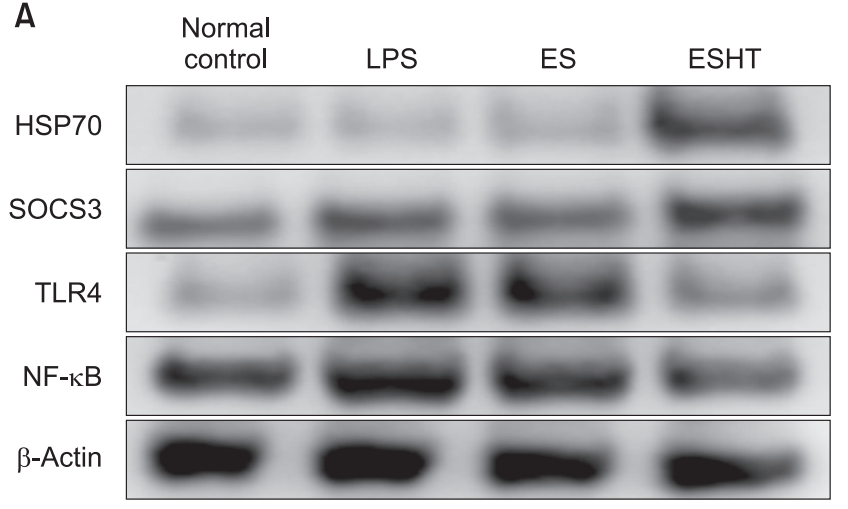

\section{B}

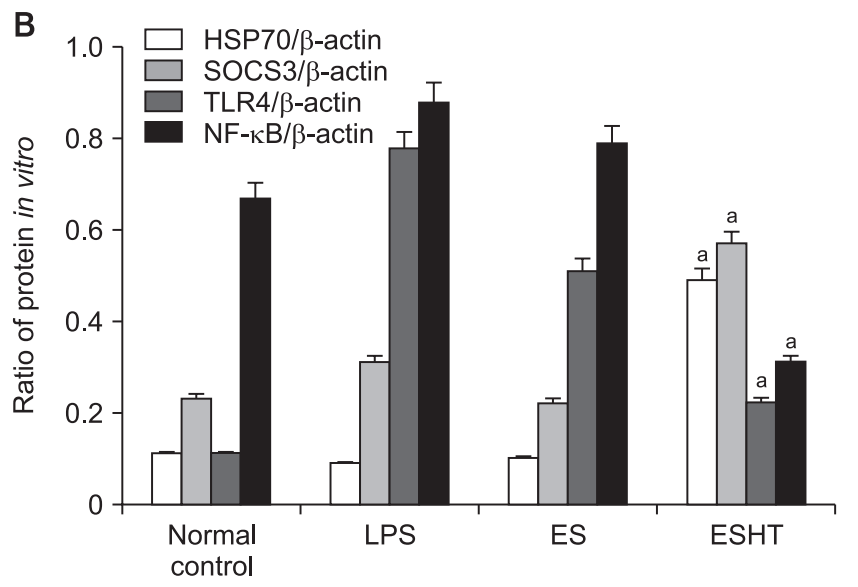

C
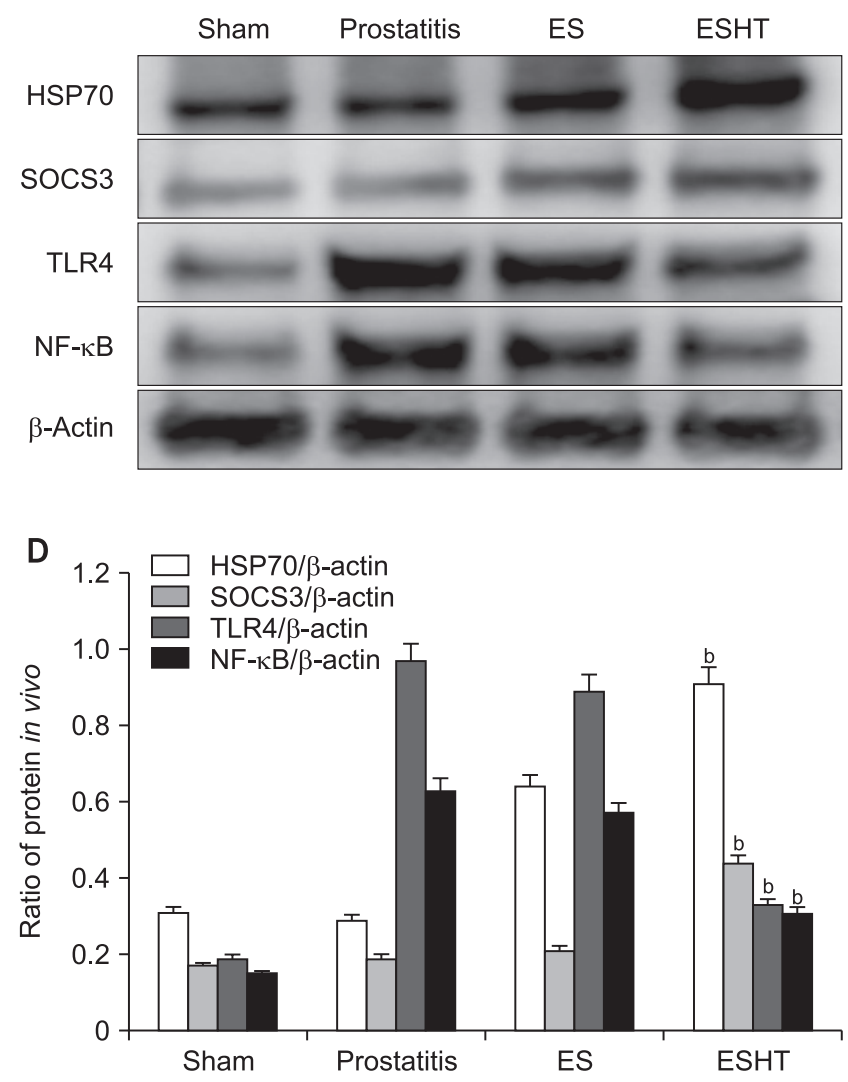

Fig. 3. Electric stimulation hyperthermia treatment (ESHT) inhibited the toll-like receptor 4 (TLR4)/nuclear factor kappa B (NF- $\kappa$ B) pathway by stimulating suppressor of cytokine signaling 3 (SOCS3) and increased HSP70 expression. (A) Western blot results of SCOS3, TLR4, HSP70, and NF$\kappa B$ in vitro for each group. (B) Quantitative analysis of Western blots for $S C O S 3 / \beta$-actin, TLR4/ $\beta$-actin, HSP70/ $\beta$-actin, and NF- $\kappa B / \beta$-actin. ${ }^{a} p<0.01$ compared to the lipopolysaccharide (LPS) group and electric stimulation (ES) group. (C) Western blot results of SCOS3, TLR4, HSP70, and NF- $\kappa B$ in vivo for each group. (D) Quantitative analysis of Western blots for SCOS3/ $\beta$-actin, TLR4/ $\beta$-actin, $\mathrm{HSP} 70 / \beta$-actin and NF- $\kappa \mathrm{B} / \beta$-actin in vivo. ${ }^{\mathrm{b}} \mathrm{p}<0.01$ compared to the prostatitis group and ES group.

\section{Electric stimulation hyperthermia treatment increased epithelial growth factor and decreased inducible nitric oxide synthase in prostatitis}

Finally, the influence of ESHT treatment on prostate tissue recovery was investigated. Fig. 5A shows EGF expression in each group. The quantitative results (Fig. $5 \mathrm{~B})$ indicate that EGF expression in the ESHT group was higher than in the other groups $(p<0.01)$. Meanwhile, to assess the magnitude of prostate tissue recovery, we detected iNOS levels in the prostate, as shown in Fig. 5C. The quantitative results in Fig. 5D indicate that iNOS levels in the ESHT group were lower $(p<0.01)$ than in the prostatitis and ES groups.

\section{DISCUSSION}

$\mathrm{CP} / \mathrm{CPPS}$ is a complicated multifactorial syndrome
[1,2], which has been treated by various treatments including drugs and physiotherapy, while the individual effects are respond to these treatments [17]. Currently, no consensus exists regarding the optimal therapy for CP/CPPS [18], making it an urgent requirement to find an effective therapy for CP/CPPS. In this study, we established a prostatitis rat model and administered ESHT as a novel treatment. We found that with ESHT, prostatitis clearly improved in the rat model. Next, we investigated the mechanism, and found that after ESHT, the expression of HPS70 and SOCS3 increased in the prostate of the rats with prostatitis, while the expression of cytokines, such as IL-1 $1 \beta$, IL-6, IL-8, and TNF- $\alpha$ decreased. These results demonstrate that the cytokines associated with inflammation are a key aspect of the effects of ESHT on prostatitis. The proposed mechanism of ESHT is shown in Fig. 6. With ESHT, levels of HSP70 and SOCS3 significantly increase, 
A

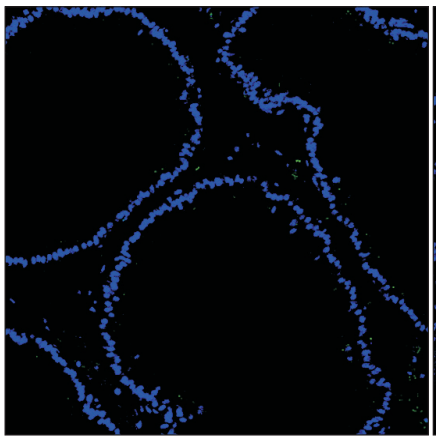

Sham

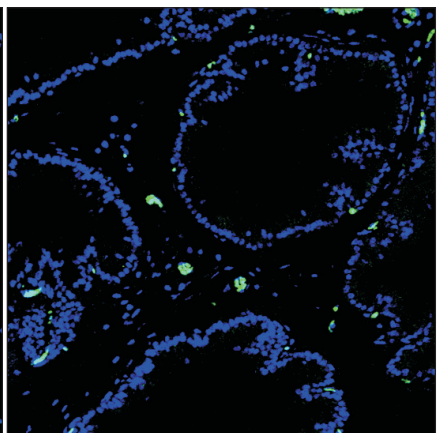

Prostatitis

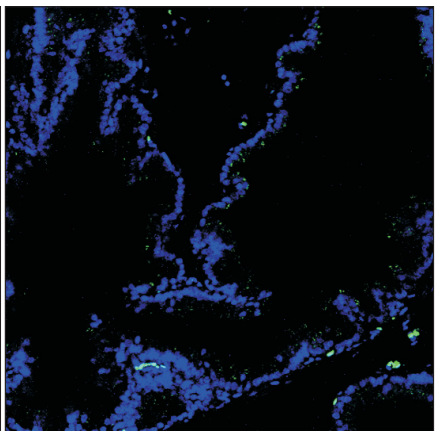

ES

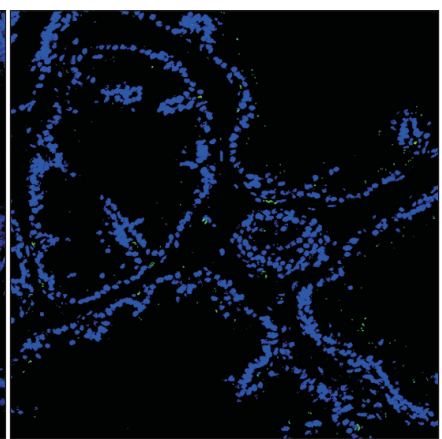

ESHT

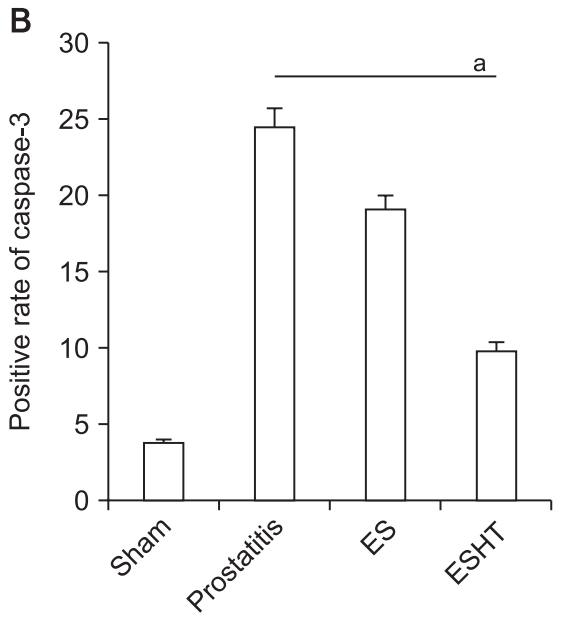

C
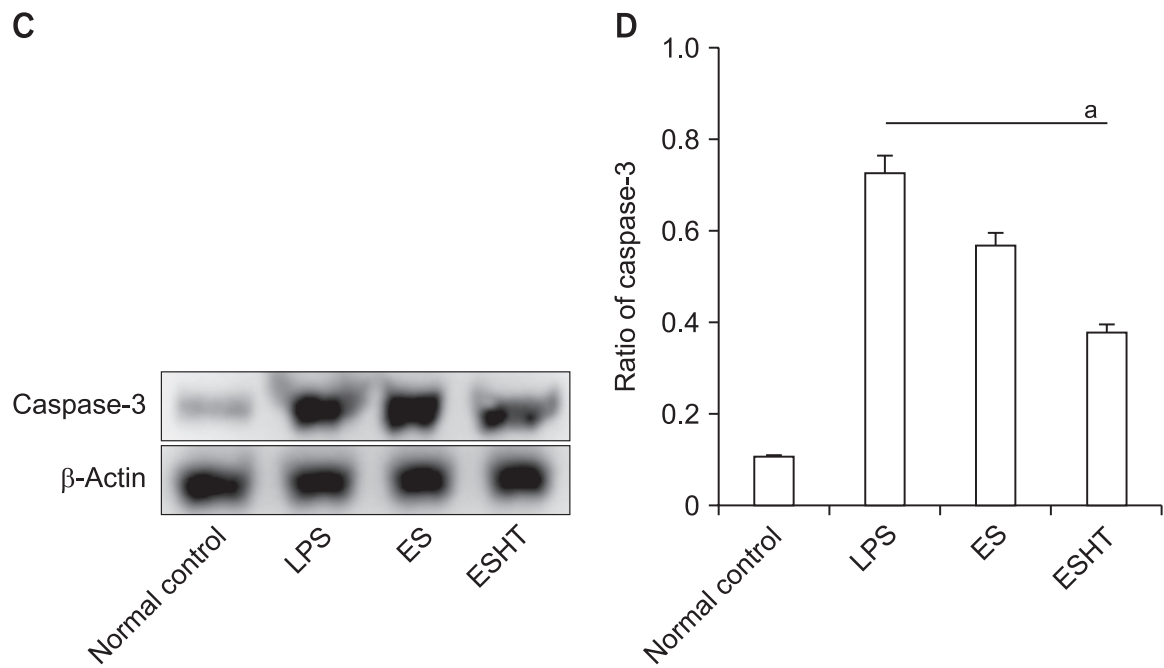

Fig. 4. Electric stimulation hyperthermia treatment (ESHT) decreased apoptosis in vivo and in vitro. (A) Representative images of caspase-3 in the prostate for each group. Green is caspase-3, blue is DAPI (4,6-diamidino-2-phenylindole). Stain method is immunohistochemistry $(\times 200)$. (B) Quantitative positive rate of caspase- 3 in vivo. ${ }^{a} p<0.01$ compared to the prostatitis group and electric stimulation (ES) group. (C) Western blot results of caspase- 3 in vitro. (D) Ratio of caspase-3/ $\beta$-actin, showing the quantitative results of the Western blot. ${ }^{a} \mathrm{p}<0.05$, compared to the lipopolysaccharide (LPS) group and ES group.

leading to binding and inhibition of the TLR4/MyD88 polymers. With the TLR4-NF- $\mathrm{B}$ pathway blocked, the expression of $\mathrm{NF}-\kappa \mathrm{B}$ also decreases. Without the regulation by $\mathrm{NF}-\kappa \mathrm{B}$ in the nucleus, inflammatory cytokines, such as IL-1 $\beta$, I-6, П-8, and TNF- $\alpha$ will not be generated by target cells. As levels of inflammatory cytokines reduce, neuroinflammation in the tissue affected by prostatitis will be mitigated, leading to expected improvements in CP/CPPS.

ES as a new and developing treatment has been applied in clinical settings for many years. Ferreira et al [19] found that the temporomandibular disorder treatment process, ES mitigated pain and improved masticatory muscle activity efficiently, without drugs or the need for invasive procedures. Rajfur et al [10] found that electrical therapy was effective in treating chronic low back pain. However, the above studies all focused on either ES alone or only on hyperthermia generated by ES, instead of studying the combination of ES and hyperthermia. Recently, Morino et al [11] found that simultaneous ES and hyperthermia treatment of diabetic mice could alleviate insulin resistance by enhancing the insulin signaling pathway; this is the first such finding, to the best of our knowledge. Patients with CP/CPPS experience anxiety about the long and uncertain treatment process, especially with invasive treatments. Therefore, ESHT is clearly a good choice as a noninvasive modality, suggesting that if it is effective, it may emerge as a preferred treatment for CP/CPPS. In this study, it was proven that ESHT improved prostatitis in a rat model. With ESHT, the pathological epithelial layer thinned and fewer papil- 
A

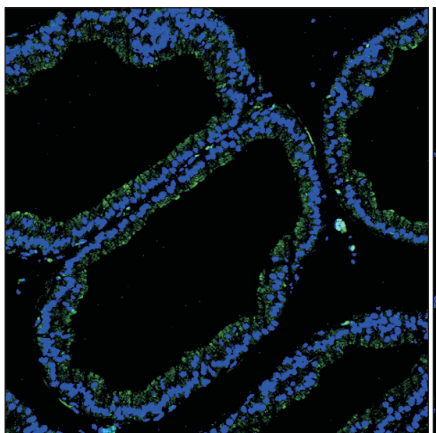

Sham

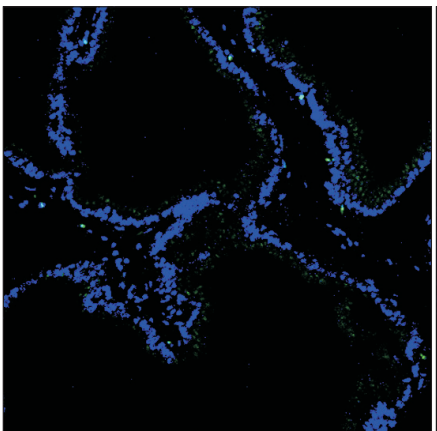

Prostatitis

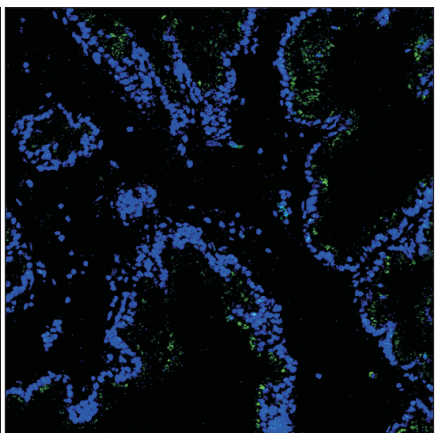

ES

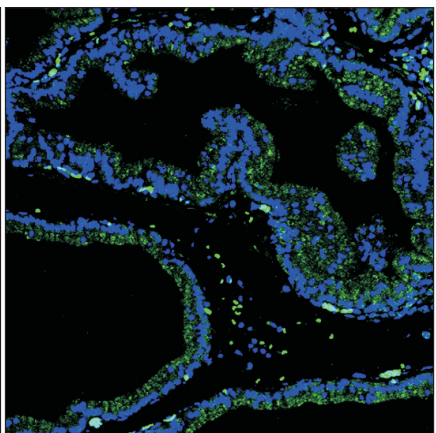

ESHT
B

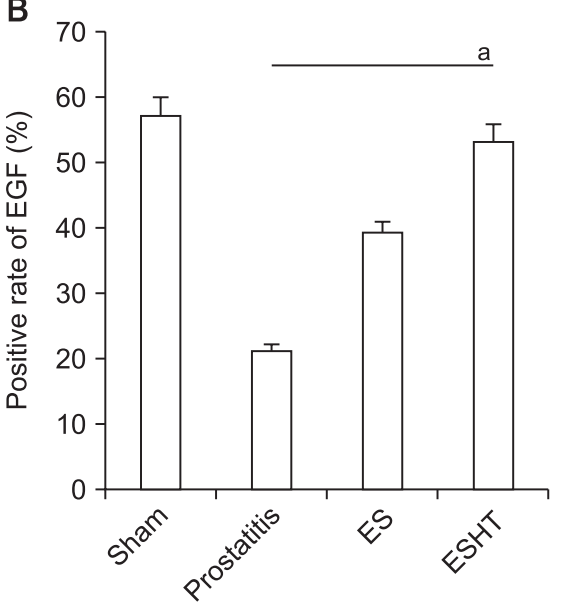

C

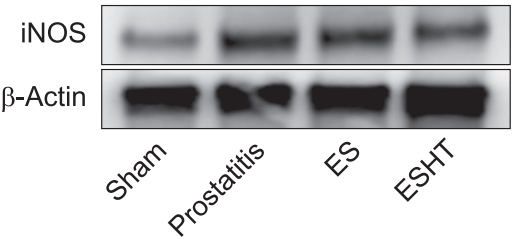

D

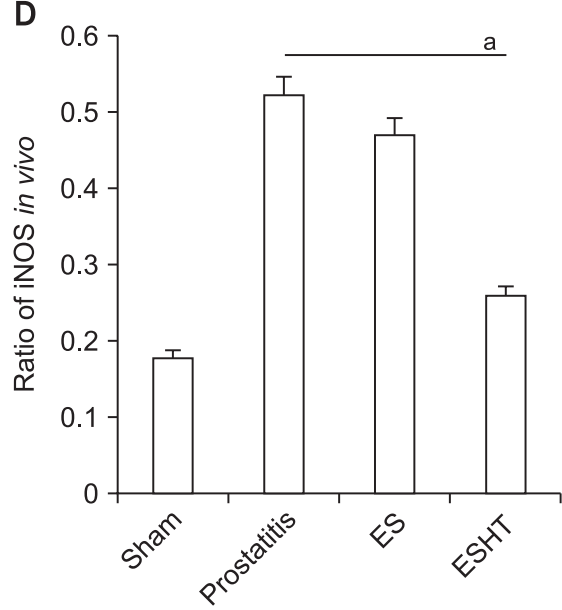

Fig. 5. Electric stimulation hyperthermia treatment (ESHT) increased epithelial growth factor (EGF) and decreased inducible nitric oxide synthase (iNOS) in vivo. (A) Representative images of EGF in the prostate for each group. Green is EGF, blue is DAPI (4,6-diamidino-2-phenylindole). Stain method is immunohistochemistry $(\times 200)$. (B) Quantitative positive rate of EGF in vivo. ${ }^{a} \mathrm{p}<0.01$ compared to the prostatitis group and electric stimulation (ES) group. (C) Western blot results for inducible nitric oxide synthase (iNOS) in vivo. (D) Ratio of iNOS/ $\beta$-actin, showing the quantitative results of the Western blot. ${ }^{a} \mathrm{p}<0.01$ compared to the prostatitis group and ES group.

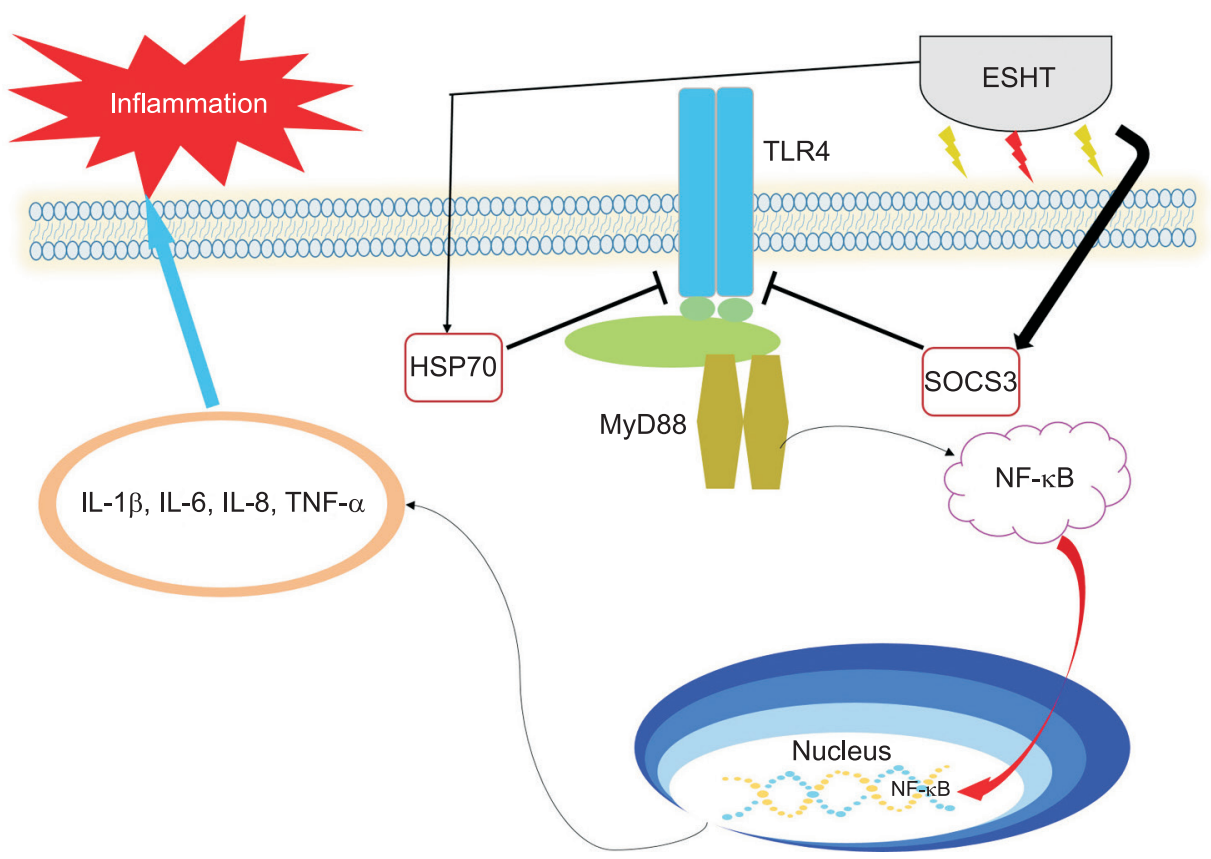

Fig. 6. The proposed mechanism through which electric stimulation hyperthermia treatment (ESHT) improves prostatitis. With ESHT, levels of HSP70 and suppressor of cytokine signaling 3 (SOCS3) significantly increase, leading to binding and inhibition of the toll-like receptor 4 (TLR4)/MyD88 polymers. With the TLR4nuclear factor kappa B (NF- $\kappa$ B) pathway blocked, the expression of NF- $\kappa B$ also decreases. Without the regulation by NF- $\kappa \mathrm{B}$ in the nucleus, inflammatory cytokines, such as interleukin (IL)-1 $\beta$, IL-6, IL-8, and tumor necrosis factor alpha (TNF- $\alpha$ ) will not be generated by target cells. 
lary fronds protruded into the glandular cavities in the prostate. In the rats with prostatitis, the expression of inflammatory factors, such as $\Pi-6, \mathbb{L}-8$, and TNF- $\alpha$ also decreased.

A recent study [5] showed neuroinflammation to be the cause of pain, as mediated by glia, with microglia in particular playing a vital role in pain sensitization. Fan et al [20] found that in postoperative pain therapy, it was possible to ameliorate postoperative pain by attenuating neuroinflammation through inducing higher levels of SOCS3 expression via the HSF1-HSP70-TLR4 pathway. Wong et al [8] also suggested that pain in $\mathrm{CP} /$ CPPS was caused by neuroinflammation. They found that prostatitis led to inflammation in the spinal cord, and that neuroinflammation and activation of microglia played an important role in the maintenance of CP/CPPS. Therefore, there are reasons to believe that ESHT would be effective in CP/CPPS treatment, as shown in this study.

HSP70 is a conditional stress protein in cells, which is expressed in great quantities in response to a short time of stimulation [21]. As an agonist of TLR4, HSP70 binds TLR4 and subsequently triggers a preliminary proinflammatory response in the cell [22]. In this experiment, we found that after ESHT, cells in the prostate expressed a large amount of HSP70. Interestingly, the expression of TLR4 decreased after HSP70 increased. Meanwhile NF- $\kappa \mathrm{B}$, a downstream protein, also decreased in the prostate tissue. As a regulatory protein, $\mathrm{NF}-\kappa \mathrm{B}$ can regulate the expression of inflammatory factors, such as $\amalg-6, \Pi-8, \mathrm{IL}-1 \beta$, and TNF- $\alpha$. Our results indicate that decreasing TLR4 reduced the expression of NF- $\mathrm{kB}$, which then diminished the expression of IL6 , IL-8, IL-1 $\beta$, and TNF- $\alpha$ in vivo. Meanwhile, it was proven that the regulation of NF- $\mathrm{B}$ by TLR4 could directly affect innate immunity and inflammation, and our results showed that SOCS3 took part in this process. Some studies have found that SOCS3 can inhibit the STAT/JAK pathway [23] and the transforming growth factor beta pathway [24], and it is also known to be a key negative regulator of the inflammatory cytokine response [25]. However, the role of SOCS3 in the TLR4 response remains controversial. Therefore, to detect the effects of SOCS3 on TLR4 more definitively, we investigated the expression of SOCS3 and TLR4 in the prostate of rats with prostatitis. We found that the expression of SOCS3 and TLR4 showed opposite trends in the prostate, meaning that SOCS3 is a nega- tive regulator of TLR4. However, we did not conduct further research on the SOCS3-TLR4 pathway to detect pathway-related factors. Speth et al [26] found that SOCS3 could affect endogenous inflammatory responses by regulating TLR4 expression. Furthermore, their report confirmed that SOCS3 is a negative regulator in the SOCS3-related pathway, which is similar to our result that SOCS3 functioned as a negative regulator of TLR4 in the SOCS3-TLR4 pathway. ESHT was administered at an early stage of prostatitis development; therefore, it is difficult to assess the implications of our findings for patients who do not receive early treatment.

\section{CONCLUSIONS}

ESHT improved CP/CPPS and reversed pathologic changes through inhibiting the SOCS3-TLR4 pathway. Our experiment also showed that HSP70 co-regulated proinflammatory factors with SOCS3 by mediating the TLR4-NF-kB pathway. It was observed that in prostatitis, SOCS3 was a master negative regulator of TLR4. This experiment may provide novel ideas for CP/CPPS treatment.

\section{ACKNOWLEDGEMENTS}

This research was supported by the Bio \& Medical Technology Development Program of the National Research Foundation (NRF) funded by the Ministry of Science \& ICT (2018M3A9E8020861).

This study was supported by Research Fund of Seoul St. Mary's Hospital, The Catholic University of Korea.

\section{Conflict of Interest}

The authors have nothing to disclose.

\section{Author Contribution}

Conceptualization: SWK, WJB. Data curation: SHJ. Formal analysis: SHJ. Funding acquisition: SWK, WJB. Investigation: GQZ. Methodology: KWL, WJT. Project administration: MKM, SHM. Resources: MKM, SHM. Software: MKM, SHM. Supervision: HJC, USH, SHH, JYL. Writing-original draft: GQZ, WJB. Writing-review \& editing: GQZ, WJB. 


\section{Data Sharing Statement}

The data analyzed for this study have been deposited in HARVARD Dataverse and are available at https://doi. org/10.7910/DVN/7D4WY3.

\section{REFERENCES}

1. Krieger JN, Nyberg L Jr, Nickel JC. NIH consensus definition and classification of prostatitis. JAMA 1999;282:236-7.

2. Zhang R, Sutcliffe S, Giovannucci E, Willett WC, Platz EA, Rosner BA, et al. Lifestyle and risk of chronic prostatitis/ chronic pelvic pain syndrome in a Cohort of United States Male Health Professionals. J Urol 2015;194:1295-300.

3. Zaidi N, Thomas D, Chughtai B. Management of chronic prostatitis (CP). Curr Urol Rep 2018;19:88.

4. Wagenlehner FM, van Till JW, Magri V, Perletti G, Houbiers JG, Weidner W, et al. National Institutes of Health Chronic Prostatitis Symptom Index (NIH-CPSI) symptom evaluation in multinational Cohorts of patients with chronic prostatitis/ chronic pelvic pain syndrome. Eur Urol 2013;63:953-9.

5. Basbaum AI, Bautista DM, Scherrer G, Julius D. Cellular and molecular mechanisms of pain. Cell 2009;139:267-84.

6. Kawasaki Y, Zhang L, Cheng JK, Ji RR. Cytokine mechanisms of central sensitization: distinct and overlapping role of interleukin-1beta, interleukin-6, and tumor necrosis factor-alpha in regulating synaptic and neuronal activity in the superficial spinal cord. J Neurosci 2008;28:5189-94.

7. Ji RR, Nackley A, Huh Y, Terrando N, Maixner W. Neuroinflammation and central sensitization in chronic and widespread pain. Anesthesiology 2018;129:343-66.

8. Wong L, Done JD, Schaeffer AJ, Thumbikat P. Experimental autoimmune prostatitis induces microglial activation in the spinal cord. Prostate 2015;75:50-9.

9. Ou J, Zhu X, Lu Y, Zhao C, Zhang H, Wang X, et al. The safety and pharmacokinetics of high dose intravenous ascorbic acid synergy with modulated electrohyperthermia in Chinese patients with stage III-IV non-small cell lung cancer. Eur J Pharm Sci 2017;109:412-8.

10. Rajfur J, Pasternok M, Rajfur K, Walewicz K, Fras B, Bolach B, et al. Efficacy of selected electrical therapies on chronic low back pain: a comparative clinical pilot study. Med Sci Monit 2017;23:85-100.

11. Morino S, Kondo T, Sasaki K, Adachi H, Suico MA, Sekimoto E, et al. Mild electrical stimulation with heat shock ameliorates insulin resistance via enhanced insulin signaling. PLoS One 2008;3:e4068.

12. Cha J, Jeon TW, Lee CG, Oh ST, Yang HB, Choi KJ, et al.
Electro-hyperthermia inhibits glioma tumorigenicity through the induction of E2F1-mediated apoptosis. Int J Hyperthermia 2015;31:784-92.

13. Kubo M, Hanada T, Yoshimura A. Suppressors of cytokine signaling and immunity. Nat Immunol 2003;4:1169-76.

14. Frobøse H, Rønn SG, Heding PE, Mendoza H, Cohen P, Mandrup-Poulsen T, et al. Suppressor of cytokine Signaling-3 inhibits interleukin-1 signaling by targeting the TRAF-6/ TAK1 complex. Mol Endocrinol 2006;20:1587-96.

15. Berlato C, Cassatella MA, Kinjyo I, Gatto L, Yoshimura A, Bazzoni F. Involvement of suppressor of cytokine signaling-3 as a mediator of the inhibitory effects of IL-10 on lipopolysaccharide-induced macrophage activation. J Immunol 2002; 168:6404-11.

16. Baetz A, Frey M, Heeg K, Dalpke AH. Suppressor of cytokine signaling (SOCS) proteins indirectly regulate toll-like receptor signaling in innate immune cells. J Biol Chem 2004;279: 54708-15.

17. Polackwich AS, Shoskes DA. Chronic prostatitis/chronic pelvic pain syndrome: a review of evaluation and therapy. Prostate Cancer Prostatic Dis 2016;19:132-8.

18. Magri V, Marras E, Restelli A, Wagenlehner FM, Perletti G. Multimodal therapy for category III chronic prostatitis/ chronic pelvic pain syndrome in UPOINTS phenotyped patients. Exp Ther Med 2015;9:658-66.

19. Ferreira AP, Costa DR, Oliveira AI, Carvalho EA, Conti PC, Costa YM, et al. Short-term transcutaneous electrical nerve stimulation reduces pain and improves the masticatory muscle activity in temporomandibular disorder patients: a randomized controlled trial. J Appl Oral Sci 2017;25:112-20.

20. Fan YX, Qian C, Liu B, Wang C, Liu H, Pan X, et al. Induction of suppressor of cytokine signaling 3 via HSF-1-HSP70TLR4 axis attenuates neuroinflammation and ameliorates postoperative pain. Brain Behav Immun 2018;68:111-22.

21. Hartl FU, Hayer-Hartl M. Molecular chaperones in the cytosol: from nascent chain to folded protein. Science 2002;295: 1852-8.

22. Doz E, Noulin N, Boichot E, Guénon I, Fick L, Le Bert M, et al. Cigarette smoke-induced pulmonary inflammation is TLR4/MyD88 and IL-1R1/MyD88 signaling dependent. J Immunol 2008;180:1169-78

23. Zhang X, Jin J, Peng X, Ramgolam VS, Markovic-Plese S. Simvastatin inhibits IL-17 secretion by targeting multiple IL17-regulatory cytokines and by inhibiting the expression of IL-17 transcription factor RORC in CD4+ lymphocytes. J Immunol 2008;180:6988-96.

24. Shirasaki T, Honda M, Shimakami T, Murai K, Shiomoto T, Okada $\mathrm{H}$, et al. Impaired interferon signaling in chronic hep- 


\section{MEN's HEALTH}

atitis $\mathrm{C}$ patients with advanced fibrosis via the transforming growth factor beta signaling pathway. Hepatology 2014;60: 1519-30.

25. Croker BA, Krebs DL, Zhang JG, Wormald S, Willson TA, Stanley EG, et al. SOCS3 negatively regulates IL-6 signaling in vivo. Nat Immunol 2003;4:540-5.
26. Speth JM, Bourdonnay E, Penke LR, Mancuso P, Moore BB, Weinberg JB, et al. Alveolar epithelial cell-derived prostaglandin E2 serves as a request signal for macrophage secretion of suppressor of cytokine signaling 3 during innate inflammation. J Immunol 2016;196:5112-20. 